\title{
Association of body mass index with risk of age-related cataracts in a middle-aged Japanese population: the JPHC Study
}

\author{
Masao Yoshida - Manami Inoue - Motoki Iwasaki • \\ Shoichiro Tsugane · JPHC Study Group
}

Received: 2 April 2010/ Accepted: 26 April 2010/Published online: 22 May 2010

(C) The Japanese Society for Hygiene 2010

\begin{abstract}
Objectives Many epidemiological studies have demonstrated that body mass index (BMI) is associated with the risk of developing age-related cataracts. These reports have suggested that high and low BMIs can affect the onset or progression of age-related visual impairment. However, few prospective studies have examined this relationship in a general Asian population. Therefore, in this study, we investigated whether BMI was associated with an increased risk of age-related cataracts by performing a 5-year prospective population-based study in a middle-aged Japanese population.

Methods This 5-year population-based study included 35,365 men and 40,825 women (aged 45-74 years), who were recruited into the Japan Public Health Center (JPHC)based Prospective Study and had not reported cataracts in a baseline survey. The self-reported diagnosis of age-related cataracts was used in the analysis of this study.

Results At follow up, 1,004 men (2.84\%) and 1,807 women $(4.43 \%)$ reported new diagnoses of age-related cataracts. The multivariate odds ratios (ORs) for those in the lowest and the highest BMI categories, compared with a BMI category of $21.0-22.9$ as a reference point (OR,
\end{abstract}

The members of the JPHC Study Group is given in the Appendix.

M. Yoshida

Department of Public Health,

Kyorin University School of Medicine,

6-20-2, Shinkawa, Mitaka, Tokyo, Japan

M. Inoue $\cdot$ M. Iwasaki $\cdot S$. Tsugane $(\varangle)$

Epidemiology and Preventive Division,

Research Center for Cancer Prevention and Screening,

National Cancer Center, 5-1-1 Tsukiji, Chuo-ku,

Tokyo 104-0045, Japan

e-mail: stsugane@ncc.go.jp
1.00), were 1.29 [95\% confidence interval (CI) 0.93-1.79] and 1.15 (95\% CI $0.96-1.39$ ) in men, and 1.23 (95\% CI $0.97-1.55)$ and 1.19 (95\% CI 1.04-1.36) in women.

Conclusions Previous studies have suggested high BMI as a risk factor of age-related cataracts for Caucasian populations in developed countries while low BMI for populations living in developing countries. In contrast to those studies, the present large-cohort study showed a U-shaped association between BMI and the incidence of cataracts in Japanese men and women.

Keywords Body mass index - Cataract - Lens opacity · Cohort study $\cdot$ Population-based

\section{Introduction}

Age-related cataract is a common eye disease, which is characterized by lens opacities and visual impairment due to the oxidation of lens proteins and degenerative changes to the lens caused by aging [1]. Cataract is a leading cause of blindness throughout the world [2-6]. The social influences of age-related cataracts are closely related to a deterioration in quality of life among the elderly. Cataract extractions, which are effective treatments for cataracts, can provide not only improvement of visual acuity but also social benefits for the patients. However, the medical care costs for cataract operations are not cheap and are a considerable drain on medical expenditure. Successful prevention of age-related cataracts is therefore thought to be important, as it could potentially lead to remarkable reductions in national medical care costs due to a decreased need for surgery [5], as well as the preservation of visual acuity for many people.

Several cohort studies have investigated various risk factors for age-related cataracts and have suggested that 
high and low body mass indexes (BMIs) are associated with increased risks of cataract occurrence in Western developed countries [7-10] and developing countries [11], respectively. However, few prospective studies have examined this relationship in a general Asian population. Although age-related cataracts are a serious problem in Japan, where the aging population is growing rapidly, a valid cohort study has not yet been performed in this country. Thus, in the present research, as a basic approach for determining the etiology of age-related cataracts, a 5-year prospective population-based study was conducted, based on the data of a cohort of 76,190 Japanese residents collected for the Japan Public Health Center (JPHC) study, in order to clarify the relationship of age-related cataracts to BMI.

\section{Subjects and methods}

\section{Study cohort}

The JPHC study is a multipurpose longitudinal cohort study of the Japanese population, which aims to investigate cancer, cardiovascular diseases, and other lifestyle-related disorders, and to obtain scientific evidence supporting effective preventive strategies for such diseases. The cohort has been divided into two groups: Cohort I, which started recruitment in 1990; and Cohort II, which started recruitment in 1993. Cohort I was composed of 54,498 residents (aged 40-59 years at baseline) with registered addresses in 14 administrative districts, who were supervised by four public health center (PHC) areas on January 1, 1990: 12,291 were from the city of Ninohe and the town of Karumai in the Ninohe PHC (Iwate Prefecture); 15,782 were from the city of Yokote and the town of Omonogawa in the Yokote PHC (Akita Prefecture); 12,219 were from eight locations (the towns of Usuda, Saku, and Koumi, and the villages of Kawakami, Minamimaki, Minami-aiki, Kita-aiki, and Yachiho) in the Minami-Saku District in the Saku PHC (Nagano Prefecture); and 14,206 were from the city of Gushikawa and the village of Onna in the Ishikawa PHC (Okinawa Prefecture). Cohort II was composed of 62,398 residents (aged 40-69 years at baseline) with registered addresses in 13 administrative districts, who were supervised by five PHC areas on January 1, 1993: 21,488 were from the town of Tomobe and Iwase in the Mito PHC (Ibaraki Prefecture); 3,571 were from the town of Oguni in the Kashiwazaki PHC (Niigata Prefecture); 8,606 were from the towns of Noichi and Kagami in the Chuo-higashi PHC (Kochi Prefecture); 14,624 were from the towns of Uku, Ojika, Shin-uonome, Arikawa, Kamigoto, and Narao in the Kamigoto PHC (Nagasaki Prefecture); and 14,109 were from the city of Hirara and town of Gusukube in the
Miyako PHC (Okinawa Prefecture). Other criteria for selecting the areas and the subjects, along with the methods of data collection and the geographic profiles of the four PHC areas for Cohort I and the five PHC areas for Cohort II, have been reported previously [12].

\section{Study participants}

In 1990 for Cohort I and in 1993 for Cohort II, a baseline self-administered questionnaire concerning demographic characteristics, medical history, lifestyle, and diet was distributed to everyone in each cohorts, and, in addition, blood samples and anthropological data were collected. In total, 43,140 individuals for Cohort I and 52,233 individuals for Cohort II completed and returned the questionnaires, giving response rates of 79 and $84 \%$, respectively.

Second and third follow-up questionnaires were sent to each participant in 1995 and 2000 for Cohort I, and in 1998 and 2003 for Cohort II, respectively. Blood samples and anthropological data were also collected in the second follow-up survey. The second follow-up questionnaires were completed and returned by 42,174 individuals for Cohort I and 50,697 individuals for Cohort II (response rates of 77 and $81 \%$ ), and 37,076 individuals (68\%) for Cohort I and 43,831 (70\%) for Cohort II also completed and returned the third follow-up questionnaires. To investigate whether age-related cataracts had occurred between the baseline and the times of the second and third surveys, we ascertained whether participants had been diagnosed with age-related cataracts, using the following question in the second and third follow-up questionnaires: "Has a doctor ever told you that you had cataracts?" As a result, a total of 2,455 participants (1,072 for Cohort I and 1,383 for Cohort II) reported that cataracts had been initially diagnosed during the first 5 years of the follow-up period. A more detailed questionnaire was then developed to investigate the history and dates of the cataract diagnoses, and the possible causes of the cataracts. In order to assess the accuracy of the answers to the questionnaire with a validation study [13], comparisons with medical records were reviewed at clinics or hospitals for 97 of the 1,072 cases in Cohort I, which were randomly selected from the 14 administrative districts of the Ninohe, Yokote, Saku, and Ishikawa PHC areas from 1998 and 1999. Of the 70 subjects who gave permission for us to review their medical records, we were able to review the records of 53 and compare them with their self-reported questionnaires. As a result, the diagnoses of age-related cataracts were confirmed in a total of 49 of the 53 self-reporters (92.5\%) [13]. This high positive-predictive value suggested that case definition from the self-reported data should be considered appropriate for use in the present study. 
To estimate the incidence of age-related cataracts during the 5-year period between the second and the third surveys (from 1995 to 2000 for Cohort I and from 1998 to 2003 for Cohort II), and to investigate the relationship between BMI and the risk of age-related cataracts, we excluded 2,455 individuals who reported a history of cataract diagnosis in the second follow-up questionnaire survey. Individuals for whom data were missing for any of the possible confounding factors described below were also excluded. After these exclusions, a total of 76,190 individuals $(35,365$ men and 40,825 women) were enrolled in this study.

This study was conducted in accordance with the Declaration of Helsinki of the World Medical Association and was approved by the Human Ethics Review Committees of the National Cancer Center of Japan.

\section{Statistical analysis}

All analyses were performed separately for men and women. An age-related cataract case was defined as a person who had been informed by an ophthalmologist that the cause of the cataract was aging. The cohort study was then conducted using an endpoint, self-reported diagnosis of age-related cataract, during the follow-up period. BMI was calculated as body weight $(\mathrm{kg})$ divided by height $(\mathrm{m})$ squared for each participant and grouped into five categories ('<19', '19.0-20.9', '21.0-22.9', '23.0-24.9', and $' \geq 25$ '). Multiple logistic-regression analyses were used to obtain the odds ratios (ORs). These were adjusted for age and the following potential confounding factors: a history of hypertension (yes or no) and diabetes (yes or no); weekly alcohol intake (g/week) using three levels of consumption in men (ALC_0 = nondrinkers and infrequent or occasional drinkers; ALC_1 $=1-299$ g/week; ALC_2 = $\geq 300 \mathrm{~g} /$ week) and in women (ALC_0 $=$ nondrinkers and infrequent or occasional drinkers; ALC_1 $=1-59 \mathrm{~g} /$ week; ALC_2 $=\geq 60 \mathrm{~g} /$ week); cigarette smoking, using three levels (nonsmokers who had never smoked, current smokers, and ex-smokers); and the PHC area. The 95\% confidence interval (CI) for each OR was calculated. The trend across increasing BMI categories was analyzed using the extended Mantel-Haenszel method. All statistical analyses were carried out using the SAS statistical software package, version 8.2 (SAS Institute, Cary, NC, USA) [14].

\section{Results}

Table 1 shows the incidence of age-related cataracts in the JPHC cohort by gender. In the 5-year follow up, there were 1,004 new diagnoses of cataracts among the men $(2.84 \%)$ and $1,807(4.43 \%)$ among the women.
Table 1 Five-year incidence of age-related cataracts in the JPHC study according to gender and PHC area

\begin{tabular}{|c|c|c|c|c|}
\hline & \multicolumn{2}{|c|}{ Cataract cases } & \multicolumn{2}{|l|}{ Total } \\
\hline & Men & Women & Men & Women \\
\hline \multicolumn{5}{|l|}{ Cohort I } \\
\hline Ninohe PHC & $91(2.30)$ & $255(5.38)$ & 3,952 & 4,829 \\
\hline Yokote PHC & $103(2.04)$ & $276(4.61)$ & 5,057 & 5,991 \\
\hline Saku PHC & $83(1.81)$ & $151(3.10)$ & 4,577 & 4,870 \\
\hline Ishikawa PHC & $72(2.31)$ & $101(2.92)$ & 3,123 & 3,453 \\
\hline Total & 349 (2.09) & $783(4.09)$ & 16,709 & 19,143 \\
\hline \multicolumn{5}{|l|}{ Cohort II } \\
\hline Mito PHC & $205(2.75)$ & $304(3.80)$ & 7,467 & 8,007 \\
\hline Kashiwazaki PHC & $35(2.93)$ & $63(4.46)$ & 1,196 & 1,353 \\
\hline Chuohigashi PHC & $104(3.80)$ & $166(5.06)$ & 2,736 & 3,279 \\
\hline Kamigoto PHC & $125(3.65)$ & $249(5.38)$ & 3,424 & 4,625 \\
\hline Miyako PHC & $186(4.85)$ & $242(5.48)$ & 3,833 & 4,418 \\
\hline Total & $655(3.51)$ & $1024(4.72)$ & 18,656 & 21,682 \\
\hline $\begin{array}{l}\text { Total (Cohort } \\
\text { I + Cohort II) }\end{array}$ & $1004(2.84)$ & $1807(4.43)$ & 35,365 & 40,825 \\
\hline
\end{tabular}

Data are cataract cases/total participants, with the percentages in parentheses

JPHC Japan Public Health Center

When the baseline characteristics of the cohort were categorized according to BMI (Table 2), a higher BMI was found to be associated with a lower age in men. The percentage of male current smokers was also inversely associated with a higher BMI. By contrast, the percentages of male nonsmokers and ex-smokers were positively associated with a higher BMI. In both sexes, the percentages of those with a history of hypertension and diabetes were positively associated with a higher BMI.

Table 3 shows the age-adjusted and multivariate ORs, with $95 \%$ CIs, for cataracts according to BMI. The results demonstrated a U-shaped association between BMI and the incidence of cataracts in both sexes. Ageadjusted ORs for those in the lowest and the highest BMI categories, compared with those in a BMI category of 21.0-22.9, were 1.13 (95\% CI $0.82-1.55)$ and 1.30 (95\% CI 1.09-1.54) in men, and 1.51 (95\% CI $1.21-$ $1.89)$ and 1.61 (95\% CI 1.42-1.83) in women. These associations remained after adjustment for age, history of hypertension and diabetes, weekly alcohol intake, cigarette smoking, and PHC area. The multivariate ORs for those in the lowest and the highest BMI categories, compared with those in a BMI category of 21.0-22.9, were 1.29 (95\% CI $0.93-1.79)$ and 1.15 (95\% CI $0.96-$ $1.39)$ in men, and 1.23 (95\% CI $0.97-1.55)$ and 1.19 (95\% CI 1.04-1.36) in women. 
Table 2 Baseline characteristics according to body mass index in middle-aged Japanese men and women a Alcohol intake (g/week ethanol), ALC_0: nondrinkers and infrequent occasional drinkers for men and women, ALC_1: 1-299 g/week for men and $1-59 \mathrm{~g} /$ week for women, ALC_2: $\geq 300 \mathrm{~g} /$ week for men and $\geq 60 \mathrm{~g} /$ week for women

\begin{tabular}{|c|c|c|c|c|c|}
\hline & \multicolumn{5}{|c|}{ Body mass index } \\
\hline & $<19.0$ & $19.0-20.9$ & $21.0-22.9$ & $23.0-24.9$ & $\geq 25.0$ \\
\hline \multicolumn{6}{|l|}{$\operatorname{Men}(n=35,365)$} \\
\hline No. of participants & 1,447 & 4,904 & 8,621 & 10,341 & 10,052 \\
\hline Age (years) & 53.6 & 52.5 & 51.8 & 51.3 & 50.7 \\
\hline \multicolumn{6}{|l|}{ Smoking status $(\%)$} \\
\hline Nonsmokers & 27.9 & 28.9 & 33.5 & 38.4 & 41.9 \\
\hline Current smokers & 58.9 & 56.6 & 50.0 & 42.8 & 38.4 \\
\hline Ex-smokers & 13.2 & 14.5 & 16.5 & 18.8 & 19.7 \\
\hline \multicolumn{6}{|l|}{ Alcohol intake ${ }^{\mathrm{a}}(\%)$} \\
\hline ALC_0 & 30.9 & 27.8 & 25.2 & 24.4 & 25.8 \\
\hline ALC_1 & 40.4 & 41.1 & 42.4 & 44.3 & 43.6 \\
\hline ALC_2 & 28.7 & 31.1 & 32.4 & 31.3 & 30.6 \\
\hline History of hypertension (\%) & 13.5 & 13.2 & 16.5 & 19.8 & 25.2 \\
\hline History of diabetes (\%) & 2.8 & 3.0 & 3.4 & 3.4 & 3.9 \\
\hline \multicolumn{6}{|l|}{ Women $(n=40,825)$} \\
\hline No. of participants & 2,202 & 5,975 & 10,484 & 10,121 & 12,043 \\
\hline Age (years) & 52.6 & 51.1 & 51.5 & 51.5 & 52.3 \\
\hline \multicolumn{6}{|l|}{ Smoking status $(\%)$} \\
\hline Nonsmokers & 91.2 & 93.1 & 94.7 & 95.2 & 94.8 \\
\hline Current smokers & 8.0 & 6.1 & 4.5 & 4.1 & 4.2 \\
\hline Ex-smokers & 0.8 & 0.8 & 0.8 & 0.7 & 1.0 \\
\hline \multicolumn{6}{|l|}{ Alcohol intake ${ }^{\mathrm{a}}(\%)$} \\
\hline ALC_0 & 83.0 & 81.5 & 81.4 & 82.3 & 85.0 \\
\hline ALC_1 & 9.7 & 11.6 & 12.0 & 11.8 & 10.1 \\
\hline ALC_2 & 7.3 & 6.9 & 6.6 & 5.9 & 4.9 \\
\hline History of hypertension (\%) & 11.4 & 11.9 & 15.4 & 19.9 & 29.4 \\
\hline History of diabetes (\%) & 1.7 & 1.4 & 1.3 & 1.7 & 2.9 \\
\hline
\end{tabular}

Table 3 Age-adjusted and multivariate odds ratios with 95\% confidence intervals for cataract diagnosis according to body mass index in middleaged Japanese men and women

Body mass index

$\begin{array}{llll}<19.0 & 19.0-20.9 & 21.0-22.9 & 23.0-24.9\end{array}$

Men

No. of cases

Age-adjusted OR $(95 \% \mathrm{CI})$

Multivariate $\mathrm{OR}^{\mathrm{a}}(95 \% \mathrm{CI})$

Women

No. of cases

Age-adjusted OR (95\% CI)

Multivariate $\mathrm{OR}^{\mathrm{a}}(95 \% \mathrm{CI})$

$\begin{array}{lll}49 & 145 & 226 \\ 1.13(0.82-1.55) & 1.08(0.87-1.33) & 1 \\ 1.29(0.93-1.79) & 1.16(0.93-1.44) & 1 \\ & & \\ 101 & 248 & 451 \\ \mathbf{1 . 5 1}(\mathbf{1 . 2 1 - 1 . 8 9 )} & \mathbf{1 . 5 1}(\mathbf{1 . 2 9 - 1 . 7 7 )} & 1 \\ 1.23(0.97-1.55) & \mathbf{1 . 2 2}(\mathbf{1 . 0 3 - 1 . 4 4 )} & 1\end{array}$

226

274

310

$.06(0.88-1.26)$

1.30 (1.09-1.54)

$1.02(0.84-1.22)$

$1.15(0.96-1.39)$

451

424

583

1.45 (1.26-1.66)

$1.61(1.42-1.83)$

$1.13(0.98-1.30)$

1.19 (1.04-1.36)

$O R$ odds ratio, $C I$ confidence intervals

${ }^{a}$ Multivariate model adjusted for age, history of hypertension and diabetes (yes or no), alcohol intake (g/week ethanol, ALC_0: nondrinkers and infrequent occasional drinkers, ALC_1: 1-299 g/week in men and 1-59 g/week in women, ALC_2: $\geq 300 \mathrm{~g} / \mathrm{week}$ in men and $\geq 60 \mathrm{~g} / \mathrm{week}$ in women), cigarette smoking (nonsmokers, current smokers, and ex-smokers), and PHC area

\section{Discussion}

Previous cohort [7-10] and cross-sectional studies [15], the Physician's Health Study [7, 9], the Framingham
Study [8], the Nurses' Health Study [15], and a prospective cohort study from the Nurses' Health Study and the Health Professionals Follow-up Study [10] have observed a higher risk of developing cataracts in people with a high 
BMI. On the contrary, in developing countries, the Lens Opacities Case-Control Study [16], the Tanjong Pager Survey [17], and the study carried out by Minassian et al. [11] found a lower BMI to be associated with increased risk of cataract occurrence. As lens opacities arise from the modification of lens proteins, in Western developed countries, which tend to have a much higher proportion of individuals with high BMI, uncontrolled glucose intake might induce cataracts by the occurrence of subclinical or clinical diabetes among the obese [15, 17]. Conversely, in individuals with a low BMI living in developing countries, malnutrition or inadequate intake of the nutrients essential to preserve lens clarity might lead to an increased risk of cataracts. Several reports [11, 18-20] have indicated a strong association of lower socioeconomic status, prevalence of diarrhea, or nutritional deficits with risk of cataracts. However, from the results of the present largecohort study, we confirmed a U-shaped association between BMI and the risk of age-related cataracts in Japanese men and women within the range of body mass usually observed.

The endpoint of the present study, the self-reported diagnosis of age-related cataract ascertained from the questionnaires, showed consecutive increases in cataract incidence with high and low BMI categories in both sexes. It is suggested that younger persons usually do not expect to have cataracts, while this expectation grows with age. After adjustment for age and multiple potential confounding factors, age-adjusted ORs, as well as multivariate ORs, were also positively associated with high and low BMI categories in both sexes. We were confident that ascertaining the occurrence of age-related cataracts from selfreporting questionnaires in this study was reliable, as we had previously carried out a study to validate this method in 1998 and 1999 [13]. The positive-predictive value for the diagnoses of age-related cataracts confirmed by medical records review was $92.5 \%$. This high positive-predictive value suggested that case definition from self-reporting questionnaires was reliable. However, there was at least one major limitation in this validation study: we were unable to evaluate the negative-predictive value of individuals who had not reported a past history of a diagnosis or extraction of cataracts.

Our study showed that the incidence of age-related cataracts was higher in women than in men. This finding is in agreement with other epidemiological studies [21]; however, an important drawback of our study is that the case definition was dependent on a self-reporting questionnaire. Underestimates of the number of cases might have occurred due to a few false-negative cases with the following origins: asymptomatic individuals who had not visited an eye clinic; individuals with undiagnosed cataract with visual impairment who had not visited an eye clinic; and diagnosed individuals who had not reported a history of the diagnoses. These sources of detection bias might have led to an underestimation of the overall incidence of age-related cataracts. Further analyses from longer follow-up studies will be required to obtain reliable evidence of the impact of BMI on the risk of age-related cataracts.

Age-related cataracts can be classified as cortical, nuclear, and posterior subcapsular (PSC) based on the opacity site. The site-specific incidences of cataracts have been assessed and analyzed in a number of recent prospective cohort $[8,10]$ and cross-sectional studies $[15,17$, 18, 22]. The Framingham Study [8], a prospective cohort study from the Nurses' Health Study and the Health Professionals Follow-up Study [10], and the Nurses' Health Study [15] have observed a higher risk of developing cortical [8], PSC [8, 10, 15], and overall cataracts [10] in people with a higher BMI. On the contrary, the Tanjong Pager Survey [17] observed a higher risk of developing cortical and overall cataracts in people with a lower BMI. Furthermore, the Salisbury Eye Evaluation project [22] observed a positive association between BMI and cortical cataracts, and an inverse association between BMI and nuclear cataracts. The Shihpai Eye Study [23] observed a U-shaped relationship between BMI and nuclear cataracts, and an inverse U-shaped relationship between BMI and cortical cataracts. In our present study, only the overall incidence could be estimated because of the use of selfreporting. However, to assess findings for the possible prevention of cataracts, the overall incidence, not sitespecific incidence, appears to be the most practical measure of evaluation in prospective population-based studies on cataracts $[9,10]$.

High and low BMIs have been suggested previously as risk factors in age-related cataracts, for Caucasian populations in Western developed countries and for the populations in developing countries, respectively; Western developed countries tend to have a much higher prevalence of obese people than Japan and developing countries have a much higher prevalence of lean individuals than Japan. In contrast to those studies, the present large-cohort study showed a U-shaped association between BMI and the incidence of age-related cataracts in Japanese men and women within the range of body mass usually observed.

Acknowledgments This study was supported by Grants-in-aid for Cancer Research and for the Third Term Comprehensive Ten-Year Strategy for Cancer Control from the Ministry of Health, Labor and Welfare of Japan. The authors thank all staff members in each study area for their efforts with the baseline and follow-up surveys. 


\section{Appendix}

Members of the Japan Public Health Center-based Prospective Study (JPHC Study, principal investigator: S. Tsugane) Group are: S. Tsugane, M. Inoue, T. Sobue, and T. Hanaoka, Research Center for Cancer Prevention and Screening, National Cancer Center, Tokyo; J. Ogata, S. Baba, T. Mannami, A. Okayama, and Y. Kokubo, National Cardiovascular Center, Osaka; K. Miyakawa, F. Satio, A. Koizumi, Y. Sano, I. Hashimoto, T. Ikuta, and Y. Tanaba, Iwate Prefectural Ninohe Public Health Center, Iwate; Y. Miyajima, N. Suzuki, S, Nagasawa, Y. Furusugi and N. Nagai, Akita Prefectural Yokote Public Health Center, Akita; H. Sanada, Y. Hatayama, F. Kobayashi, H. Uchino, Y. Shirai, T. Kondo, R. Sasaki, Y. Watanabe, Y. Miyagawa, and Y. Kobayashi, Nagano Prefectural Saku Public Health Center, Nagano; Y. Kishimoto, E. Takara, T. Fukuyama, M. Kinjo, M. Irei, and H. Sakiyama, Okinawa Prefectural Chubu Public Health Center, Okinawa; K. Imoto, H. Yazawa, T. Seo, A. Seiko, F. Ito, and F. Shoji, Katsushika Public Health Center, Tokyo; A. Murata, K. Minato, K. Motegi, and T. Fujieda, Ibaraki Prefectural Mito Public Health Center, Ibaraki; K. Matsui, T. Abe, M. Katagiri, and M. Suzuki, Niigata Prefectural Kashiwazaki and Nagaoka Public Health Center, Niigata; M. Doi, A. Terao, Y. Ishikawa, and T. Tagami, Kochi Prefectural Chuo-higashi Public Health Center, Kochi; H. Sueta, H. Doi, M. Urata, N. Okamoto, and F. Ide, Nagasaki Prefectural Kamigoto Public Health Center, Nagasaki; H. Sakiyama, N. Onga, H. Takaesu, and M. Uehara, Okinawa Prefectural Miyako Public Health Center, Okinawa; F. Horii, I. Asano, H. Yamaguchi, K. Aoki, S. Maruyama, M. Ichii, and M. Takano, Osaka Prefectural Suita Public Health Center, Osaka; Y. Tsubono, Tohoku University, Miyagi; K. Suzuki, Research Institute for Brain and Blood Vessels Akita, Akita; Y. Honda, K. Yamagishi, S. Sakurai, and N. Tsuchiya, Tsukuba University, Ibaraki; M. Kabuto, National Institute for Environmental Studies, Ibaraki; M. Yamaguchi, Y. Matsumura, S. Sasaki, and S. Watanabe, National Institute of Health and Nutrition, Tokyo; M. Akabane, Tokyo University of Agriculture, Tokyo; T. Kadowaki, Tokyo University, Tokyo; M. Noda and T. Mizoue, International Medical Center of Japan, Tokyo; Y. Kawaguchi, Tokyo Medical and Dental University, Tokyo; Y. Takashima and M. Yoshida, Kyorin University, Tokyo; K. Nakamura, Niigata University, Niigata; S. Matsushima and S. Natsukawa, Saku General Hospital, Nagano; H. Shimizu, Sakihae Institute, Gifu; H. Sugimura, Hamamatsu University, Shizuoka; S. Tominaga, Aichi Cancer Center Research Institute, Aichi; H. Iso, Osaka University, Osaka; M. Iida, W. Ajiki, and A. Ioka, Osaka Medical Center for Cancer and Cardiovascular Disease, Osaka; S. Sato, Osaka Medical Center for Health Science and Promotion, Osaka; E. Maruyama, Kobe University, Hyogo; M. Konishi, K. Okada, and I. Saito, Ehime University, Ehime; N. Yasuda, Kochi University, Kochi; S. Kono, Kyushu University, Fukuoka.

\section{References}

1. Jacques PF, Taylor A. Micronutrients and age-related cataracts. In: Bendich A, Buttterworth CE Jr, editors. Micronutrients in health and in disease prevention. New York: Marcel Dekker; 1991. p. 359-79.

2. Kupfer C. Bowman lecture. The conquest of cataract: a global challenge. Trans Ophthalmol Soc U K. 1985;104:1-10.

3. Kupfer C, Underwood B, Gillen T. Leading causes of visual impairment worldwide. In: Albert DM, Jakobiec FA, editors. Principles and practice of ophthalmology: basic sciences. Philadelphia: WB Saunders; 1994. p. 1249-55.

4. Leske MC, Wu SY, Hyman L, Nemesure B, Hennis A, Schachat AP, Barbados Eye Studies Group. Four-year incidence of visual impairment: Barbados Incidence Study of Eye Disease. Ophthalmology. 2004;111:118-24.

5. Foster A. Cataract-a global perspective: output, outcome and outlay. Eye. 1999;13:449-53.

6. Dunzhu S, Wang FS, Countright P, et al. Blindness and eye diseases in Tibet: findings from a randomized, population based survey. Br J Ophthalmol. 2003;87:1443-8.

7. Glynn RJ, Christen WG, Manson JE, Bernheimer J, Hennekens $\mathrm{CH}$. Body mass index. An independent predictor of cataract. Arch Ophthalmol. 1995;113:1131-7.

8. Hiller R, Podger MJ, Sperduto RD, et al. A longitudinal study of body mass index and lens opacities. The Framingham Studies. Ophthalmology. 1998;105:1244-50.

9. Schaumberg DA, Glynn RJ, Christen WG, Hankinson SE, Hennekens $\mathrm{CH}$. Relations of body fat distribution and height with cataract in men. Am J Clin Nutr. 2000;72:1495-502.

10. Weintraub JM, Willett WC, Rosner B, Colditz GA, Seddon JM, Hankinson SE. A prospective study of the relationship between body mass index and cataract extraction among US women and men. Int J Obes Relat Metab Disord. 2002;26:1588-95.

11. Minassian DC, Mehra VJ, Jones BR. Dehydrational crises from severe diarrhoea or heatstroke and risk of cataract. Lancet. 1984;1:751-3.

12. Tsugane S, Sobue T. Baseline survey of JPHC study-design and participation rate. Japan Public Health Center-based Prospective Study on Cancer and Cardiovascular Diseases. J Epidemiol. 2001;11(suppl):S24-9.

13. Takashima Y, Yoshida M, Yoshinaga A, Sasaki S, Tsugane S, JPHC Study Group. Usefulness of the recall-based self-report as a means of case definition in epidemiological studies on senile cataract. Jpn J Health Hum Ecol. 2002;68:43-53.

14. SAS Institute Inc. SAS/STAT user's guide, Version 8.2. Cary, North Carolina: SAS Institute Inc.; 1999.

15. Jacques PF, Moeller SM, Hankinson SE, et al. Weight status, abdominal adiposity, diabetes, and early age-related lens opacities. Am J Clin Nutr. 2003;78:400-5.

16. Leske MC, Chylack LT Jr, Wu SY. The Lens Opacities CaseControl Study. Risk factors for cataract. Arch Ophthalmol. 1991;109:244-51.

17. Foster PJ, Wong TY, Machin D, Johnson GJ, Seah SK. Risk factors for nuclear, cortical and posterior subcapsular cataracts in the Chinese population of Singapore: the Tanjong Pager Survey. Br J Ophthalmol. 2003;87:1112-20. 
18. Jacques PF, Chylack LT Jr, McGandy RB, Hartz SC. Antioxidant status in persons with and without senile cataract. Arch Ophthalmol. 1988;106:337-40.

19. The Italian-American Cataract Study Group. Risk factors for agerelated cortical, nuclear, and posterior subcapsular cataracts. Am J Epidemiol. 1991;133:541-53.

20. Hankinson SE, Stampfer MJ, Seddon JM, et al. Nutrient intake and cataract extraction in women: a prospective study. BMJ. 1992;305:335-9.
21. Lesk MC, Sperduto RD. The epidemiology of senile cataracts: a review. Am J Epidemiol. 1983;118:152-65.

22. Caulfield LE, West SK, Barron Y, Cid-Ruzafa J. Anthropometric status and cataract: the Salisbury Eye Evaluation Project. Am J Clin Nutr. 1999;69:237-42.

23. Kuang TM, Tsai SY, Hsu WM, Cheng CY, Liu JH, Chou P. Body mass index and age-related cataract: the Shihpai Eye Study. Arch Ophthalmol. 2005;123:1109-14. 